Après de longs travaux préparatoires et des négociations d'une incroyable âpreté, le véritable fondateur de «TARMED Suisse SA", notre vice-président, le Dr Ernst Gähler, aurait eu beaucoup de plaisir à vous révéler que la voie est enfin libre pour la révision de la structure tarifaire ambulatoire. En lieu et place, c'est avec une immense tristesse que nous vous communiquons le décès de notre estimé collègue Ernst Gähler survenu subitement le 12 mars 2015 d'une défaillance cardiaque. Avec Ernst, nous perdons une personnalité extraordinaire, un ami et un homme remarquable, qui a fait preuve d'un engagement sans faille au service des patients et des médecins.

Dr Jürg Schlup, président de la FMH

\title{
Première pierre de la nouvelle structure tarifaire ambulatoire
}

La société «TARMED Suisse SA» a été fondée aujourd'hui à Berne. Son rôle est de poursuivre le développement et d'actualiser la structure tarifaire TARMED pour les traitements médicaux ambulatoires dans les cabinets et les hôpitaux. La structure tarifaire TARMED révisée doit être prête pour la fin 2015, avant d'être soumise au Conseil fédéral pour approbation et d'entrer en vigueur début 2017.

En fondant la société «TARMED Suisse SA», les quatre partenaires tarifaires - curafutura, FMH, H+ et CTM créent au niveau organisationnel les conditions d'une révision complète et d'une poursuite du développement de TARMED, le tarif des prestations médicales ambulatoires en cabinet et à l'hôpital. L'objectif de la nouvelle organisation consiste à mettre en place une tarification professionnelle avec un bureau tarifaire autonome. Sur mandat des partenaires, «TARMED Suisse SA» assurera le suivi de la structure et l'adaptera en continu aux évolutions médicales et économiques. La société dispose des compétences nécessaires pour tenir à jour le tarif et actualiser les bases de calcul. Cette démarche professionnelle ouvre la voie à de nouvelles structures tarifaires appropriées, qui respectent les critères économiques et correspondent à l'activité médicale.

Les partenaires contractuels revendiquent la séparation entre prix et structure. Cela signifie que «TARMED Suisse SA» se limitera à élaborer et à poursuivre le développement d'une structure tarifaire qui reflète l'activité, recense les prestations médicales et les évalue sous forme de points. Les valeurs du point, qui détermineront le montant de la rémunération d'une prestation, continueront d'être négociées entre les fournisseurs de prestations et les assureurs.

La séparation stricte entre les travaux menés sur la structure et les négociations des prix doit permettre de surmonter le blocage déjà ancien de l'évolution de TARMED. Une structure appropriée et adaptée aux règles de l'économie est aussi bien dans l'intérêt des partenaires tarifaires nationaux que dans celui des patients et des payeurs de primes.

Dans le cadre de la révision globale de TARMED, les cofondateurs s'efforceront en outre de simplifier la structure afin de rendre la facturation plus transparente et compréhensible. La structure tarifaire TARMED révisée doit être prête pour la fin 2015, avant d'être soumise au Conseil fédéral pour approbation et d'entrer en vigueur début 2017.

L'assemblée de fondation de "TARMED Suisse SA» a élu Walter Bosshard en tant que président externe. Le conseil d'administration est en outre composé paritairement de représentants des secteurs des fournisseurs de prestations et des assureurs.

"TARMED Suisse SA» regroupe la Fédération des médecins suisses FMH, l'association des hôpitaux H+, l'association d'assureurs curafutura ainsi que la Commission des tarifs médicaux CTM, réunissant les assureurs accidents, auxquels sont également rattachées l'assurance militaire et l'assurance invalidité. Les membres fondateurs sont ouverts à la négociation avec d'autres partenaires tarifaires pour conclure des conventions d'utilisation de la structure révisée ou pour intégrer d'autres associations d'assureurs à «TARMED Suisse SA». 